\title{
Enantiomeric resolution of helicochiral paddlewheel complexes and their infrared, Raman, UV-vis and X-ray optical activity
}

E. A. Hillard,${ }^{a}$ T. Buffeteau, ${ }^{b}$ V. Bulicanu, ${ }^{a}$ R. Clérac, ${ }^{a}$ M. Cortijo, ${ }^{a, c}$ N. Daugey, ${ }^{b}$ A. Naim, ${ }^{c}$ A. Rogalev, ${ }^{d}$ P. Rosa, ${ }^{c}$ P. Sainctavit, ${ }^{e}$ A. Srinivasan, ${ }^{a}$ A. Valentín-Pérez, ${ }^{a, c}$ F. Wilhelm ${ }^{d}$

${ }^{a}$ CNRS, Univ. Bordeaux, CRPP, UMR 5031, 115 Avenue Schweitzer, 33600 Pessac, France

${ }^{b}$ CNRS, Univ. Bordeaux, ISM, UMR 5255, 351 Cours de la Libération, 33405 Talence, France

${ }^{c}$ CNRS, Univ. Bordeaux, ICMCB, UMR 5026, 87 Avenue Schweitzer, 33600 Pessac, France

${ }^{c}$ European Synchrotron Radiation Facility (ESRF), 38043 Grenoble, France

${ }^{d}$ CNRS, Univ. Sorbonne, IMPMC, UMR 7590, 4 place Jussieu, 75252 Paris Cedex 05, France

E-mail: hillard@crpp-bordeaux.cnrs.fr

Linear polynuclear paddlewheel complexes - "extended metal atom chains" or "metal strings" - have provided attractive models for the study of metal-metal bonding, magnetism and conductivity since their discovery in the 1990s [1]. Their helicoidal chirality, arising from mutual steric hindrance of the 3-pyridyl protons, resulting in the twisting of the equatorial ligand around the metal axis (see figure), has been less studied. Nonetheless, in one of the few examples of chiral resolution, the obtained enantiomers of a trinickel complex showed a remarkably high specific rotation of $\pm 5000 \mathrm{deg} \cdot \mathrm{mL} \cdot \mathrm{g}^{-1} \cdot \mathrm{dm}^{-1}[2]$, motivating us to seek a general technique for the chiral resolution of such racemates.

We have developed a procedure based on anion exchange for the chiral resolution of $\left[\mathrm{M}_{3}(\mathrm{dpa})_{4}\right]^{2+}$
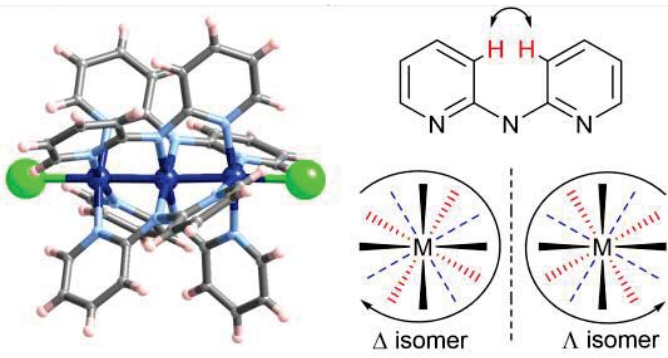

Chart 1. (Left) Crystallographic model of $\left[\mathrm{Co}_{3}(\mathrm{dpa})_{4} \mathrm{Cl}_{2}\right]$; cobalt (dark blue), nitrogen (pale blue), chloride (green). (Right) Helicoidal wrapping of dpa ligand; arrow indicates hydrogen atom repulsions. salts $\left(\mathrm{M}=\mathrm{Co}(\mathrm{II})\right.$ or $\mathrm{Ni}(\mathrm{II})$, Hdpa $=2,2^{\prime}$ dipyridylamine). Homochiral arsenyl tartrate (AsT) salts promoted the selective crystallization of $\left[\Delta-\mathrm{M}_{3}(\mathrm{dpa})_{4}(\mathrm{MeCN})_{2}\right]\left(\mathrm{NBu}_{4}\right)_{2}[\Lambda-\mathrm{AsT}]_{2}$, or $\left[\Lambda-\mathrm{M}_{3}(\mathrm{dpa})_{4}(\mathrm{MeCN})_{2}\right]\left(\mathrm{NBu}_{4}\right)_{2}[\Delta-\mathrm{AsT}]_{2}$ in the $P 42{ }_{1} 2$ space group. The enantiopure compounds demonstrated surprisingly large optical activities using UV-vis, Raman and infrared spectroscopy in solution and, for the cobalt derivatives, in the $\mathrm{X}$-ray range at the Co K-edge in single crystals. An intense X-ray linear dichroism was observed in the orthoaxial crystal orientation, whereas it vanished in the axial confirmation, while the angular dependence of the circular dichroism spectra followed the expected $\left(3 \cos ^{2} \theta-1\right)$ function, thus spectroscopically confirming the $D_{4}$ crystal symmetry. X-ray magnetic circular dichroism and X-ray magnetochiral dichroism signals at the Co K-edge were not detected, likely due to a strongly delocalized spin density on the metal-metal bonded tricobalt core. Nevertheless, these results establish that chiral polynuclear paddlewheel complexes can be cleanly resolved using selective crystallization and demonstrate considerable optical activity in the infrared, UV-vis and X-ray energy ranges, thus potentially offering future perspectives in non-linear optics and asymmetric synthesis [3].

\section{Acknowledgements}

Financial Support: CNRS, Univ. Bordeaux, the Conseil Régional de la Nouvelle Aquitaine, the France Canada Research fund (A. S.), the European Union's Horizon 2020 research and innovation program under the Marie Sklodowska-Curie grant agreement $N^{\circ} 706556$ CHIMMM (M. C.), the Erasmus Mundus European program (V. B.), the ANR project CHIROTS ANR-11-JS07-013-01 (P. R. and A. N.), and the European Synchrotron Radiation Facility. The authors warmly thank L. Falvello, K. Ollefs, F. Guillou, A. Hen, P. Voisin, M. Rouzières, L. Etienne and D. Denux for technical assistance and useful discussions.

[1] J. F. Berry, in Multiple Bonds between Metal Atoms, F. A. Cotton, C. A. Murillo, R. A. Walton, eds. Springer U.S., 2005, pp. 669-706.

[2] M. M. Warnke, F. A. Cotton, D. W. Armstrong, Chirality 2007, 19, 179.

[3] A. Srinivasan, M. Cortijo, V. Bulicanu, A. Naim, R. Clérac, P. Sainctavit, A. Rogalev, F. Wilhelm, P. Rosa,

E. A. Hillard, Chem. Sci. 2018, 9, 1136. 